\title{
Portal Vein Thrombosis-a Rare Complication of SARS-CoV-2 Infection
}

\author{
Neeraj Sharma ${ }^{1}$ (D) $\cdot$ Rajat Shukla $^{1} \cdot$ Kunal Kumar $^{1} \cdot$ Sumeet Arora ${ }^{1} \cdot$ Rachna Warrier $^{1} \cdot$ Subin Philip $^{2}$
}

Accepted: 23 March 2021 / Published online: 7 April 2021

(C) The Author(s), under exclusive licence to Springer Nature Switzerland AG 2021

\begin{abstract}
The SARS-CoV-2 is the causative organism for COVID-19 disease. It primarily affects the respiratory system. With time, some new extra-pulmonary manifestations of COVID-19 disease have been identified. Recent studies have shown that patients with SARS-CoV-2 infection may have a hypercoagulable state which explains the increased incidence of thrombotic events in these patients without any known risk factors. The most common thrombotic event described in these patients is pulmonary embolism. Intra-abdominal thrombosis is a rare thrombotic complication of COVID-19 disease. Here, we report a case of COVID-19 disease associated with acute portal vein thrombosis.
\end{abstract}

Keywords SARS-CoV-2 $\cdot$ COVID-19 $\cdot$ Thromboembolism $\cdot$ Portal vein thrombosis

\section{Introduction}

The SARS-CoV-2 is the causative organism for COVID-19 disease. It primarily affects the respiratory system. The clinical presentation of this disease varies from a mild flu-like syndrome to a severe viral pneumonia with acute respiratory insufficiency [1]. With time, some new extra-pulmonary manifestations of COVID-19 disease have been identified. Recent studies have shown that patients with SARS-CoV-2 infection may have a hypercoagulable state which explains the

\begin{tabular}{l}
\hline This article is part of the Topical Collection on Covid-19 \\
\hline $\begin{array}{l}\text { Neeraj Sharma } \\
\text { dmt18sharma@gmail.com }\end{array}$ \\
Rajat Shukla \\
docrajatshukla@gmail.com \\
Kunal Kumar \\
kunalgutgutia@gmail.com \\
Sumeet Arora \\
drsumeetarora@ hotmail.com \\
Rachna Warrier \\
rachnawarrier@gmail.com \\
Subin Philip \\
subin4philip@gmail.com \\
Military Hospital Namkum, Ranchi, Jharkand 834010, India \\
$2 \quad$ Military Hospital Ramgarh, Jharkand, India
\end{tabular}

increased incidence of thrombotic events in these patients without any known risk factors. The most common thrombotic event described in these patients is pulmonary embolism [2]. Intra-abdominal thrombosis is a rare thrombotic complication of COVID-19 disease. Here, we report a case of COVID-19 disease associated with portal vein thrombosis.

\section{Case Presentation}

The patient was a 28 -year-old male with no known comorbidities. He was admitted with chief complaint of worsening colicky abdominal pain over epigastric and umbilical region of 3 days duration. It was associated with nausea, vomiting, and constipation. He was a non-smoker and used to consume alcohol occasionally (twice a month). He denied history of abdominal trauma and similar complaints in past. On examination, he appeared dehydrated. He had tachycardia (110/min) with normal oxygen saturation of $98 \%$ on room air and normal respiratory rate. Abdominal examination revealed severe epigastric and umbilical tenderness with guarding. Laboratory workup on admission is summarized in Table 1. It showed total white blood cell count (WBC) $10,400 \mu / \mathrm{L}$ with predominant neutrophils, urea $16 \mathrm{mg} / \mathrm{dl}$, creatinine $1.0 \mathrm{mg} / \mathrm{dl}$, aspartate aminotransferase (AST) $38 \mathrm{U} / \mathrm{L}$, alanine aminotransferase (ALT) $86 \mathrm{U} / \mathrm{L}$, serum lipase $313 \mathrm{U} / \mathrm{L}$, serum amylase $75 \mathrm{U} / \mathrm{L}$, and total bilirubin of $0.8 \mathrm{mg} / \mathrm{dl}$ (Table 1). During hospital stay, his nasopharyngeal swab test for COVID-19 RT-PCR was 
Table 1 Laboratory workup on admission

\begin{tabular}{ll} 
Total leucocyte count $\left(\right.$ per $\left.\mathrm{mm}^{3}\right)$ & 10,400 \\
Differential leucocyte count & $\mathrm{N}-85 \%, \mathrm{~L}-12 \%, \mathrm{M}-1 \%, \mathrm{E}-2 \%$ \\
Platelet count (per $\left.\mathrm{mm}^{3}\right)$ & 3.12 lakh \\
Hemoglobin (g/dl) & 13.6 \\
Hematocrit & $43.5 \%$ \\
Total bilirubin (mg/dl) & 0.8 \\
ALT (U/L) & 86 \\
AST (U/L) & 38 \\
Lactate dehydrogenase (U/liter) & 421 \\
Urea (mg/dl) & 16 \\
Creatinine (mg/dl) & 1.0 \\
Amylase (U/L) & 75 \\
Lipase (U/L) & 313 \\
CRP & Positive \\
D-dimer (ng/ml) & $1533(0-500)$ \\
Ferritin (ng/ml) & $410(0-322)$ \\
SARS-CoV-2 (nasopharyngeal swab) & RT-PCR positive \\
Procalcitonin (ng/ml) & 0.46 \\
Protein C & $92(70-106 \%)$ \\
Protein S & $64(55-160 \%)$ \\
Antithrombin III (g/l) & $0.33(0.22-0.39)$ \\
Cardiolipin antibodies (IgG) & $7.21(<10)$ \\
Factor VIII & $95(70-150 \%)$ \\
ANA & Negative \\
P ANCA/C ANCA & Negative \\
\hline & \\
\hline
\end{tabular}

done (hospital protocol due to ongoing COVID-19 pandemic) which came out to be positive. The chest radiograph was normal. He underwent ultrasound of the abdomen which showed echogenic debris in right and left branches of portal vein consistent with thrombus and mild hepatosplenomegaly with ascites. He was subjected to contrast-enhanced computed tomography (CECT) of abdomen which showed portal and superior mesenteric vein thrombosis with mild hepatomegaly with minimal ascites (Fig. 1). His inflammatory markers (CRP, LDH, ferritin) were also raised. He was subjected to thrombophilia workup (antithrombin III, lupus anticoagulant, protein $\mathrm{C}$, protein $\mathrm{S}$, anti-nuclear antibody (ANA), antineutrophil cytoplasmic antibody (ANCA), factor V Leiden, and prothrombin G20210A mutations) which came out to be normal. Flow cytometric testing for paroxysmal nocturnal hemoglobinuria was also negative. Hence after ruling out inherited/acquired causes of thrombophilia and the local predisposing factors (liver cirrhosis, pancreatitis, diverticulitis, and cholecystitis), his final diagnosis was acute portal vein thrombosis secondary to COVID-19 disease. He was managed in intensive care unit with parenteral empirical antibiotics, inj low molecular weight heparin (LMWH), IV fluids, and bowel rest. He responded well to the therapy and was later switched to oral novel anticoagulant agent (apixaban, $5 \mathrm{mg}$ BD). He underwent upper gastrointestinal endoscopy (UGIE) later to rule out varices and was found to be normal (Fig. 2). He will remain anticoagulated for a minimum of 6 months and was advised to review after 3 months of anticoagulation therapy.

\section{Discussion}

The clinical presentation of SARS-CoV-2 infection seems to be variable, including asymptomatic infection, mild upper respiratory infection, and severe pneumonia with respiratory failure [3]. The involvement of gastrointestinal system in COVID-19 disease has been reported in an increasing number of patients with symptoms such as vomiting, diarrhea, nausea, and abdominal pain. The largest meta-analysis of gastrointestinal symptoms associated with COVID-19 infection found $18 \%$ of patients presented with gastrointestinal symptoms, with around 9.2\% developing abdominal pain [4].

Portal vein thrombosis is a rare cause of acute abdominal pain in both children and adults. It occurs in association with a wide variety of precipitating factors which include diseases such as decompensated liver cirrhosis and/or malignancies, pancreatitis, systemic lupus erythematosus, and other hypercoagulable states [5]. COVID-19 disease has been associated with a hypercoagulable state which can predispose to thrombotic events [6]. The incidence of thromboembolic events in the setting of COVID-19 infection overall is not known. However, in the intensive care setting with critically ill patients, thrombotic complications related to COVID-19 are reported in $25-31 \%$ of patients. The majority of thromboembolic events were pulmonary emboli, deep venous thrombosis (DVT), and cerebral infarction usually occurring in the elderly with severe COVID-19 and comorbid diseases such as diabetes and hypertension $[7,8]$. In our case, the patient was a young man without any known comorbidities. The intra-abdominal thrombosis is a rare complication related to COVID-19 infection and is sparsely reported in the literature. There are few case reports on acute portal vein thrombosis in COVID-19 patient [9-11]. Jafari et al. reported the abdominal CT findings of a 26-year-old male patient with COVID-19 who had severe abdominal pain during hospitalization and was later diagnosed with portal vein thrombosis [12]. Borazjani et al. also reported a 23-year-old asthmatic male with coronavirus pneumonia who developed acute generalized abdominal pain. Further evaluations revealed a portal vein thrombosis and mild ascites although the patient received proper anticoagulation therapy. Routine lab investigations regarding the secondary causes of portal vein thrombosis were normal [13]. In our patient, inherited and secondary causes for hypercoagulable state and the local 


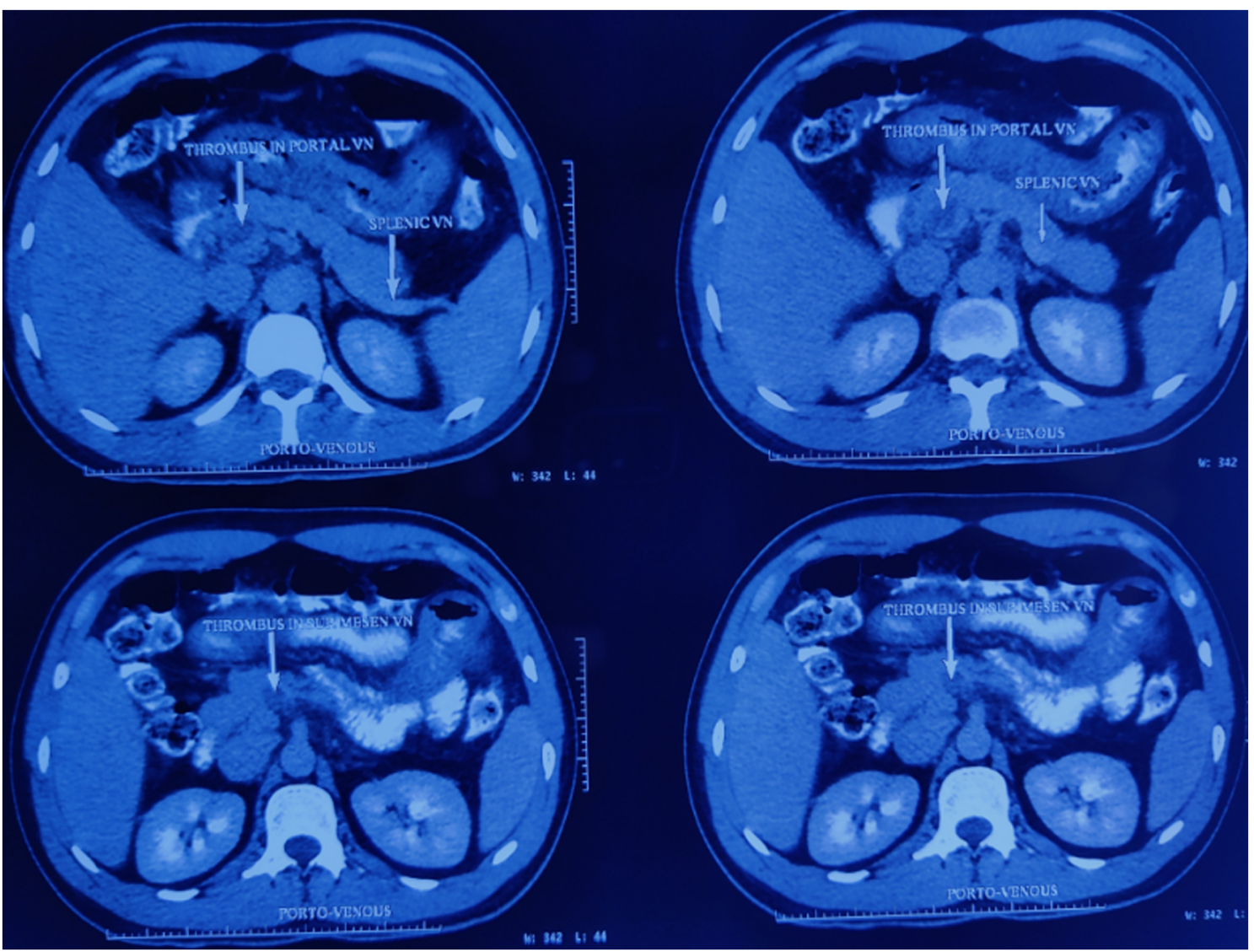

Fig. 1 Contrast-enhanced computed tomography of abdomen showing portal vein thrombosis

predisposing factors (liver cirrhosis, pancreatitis, diverticulitis, and cholecystitis) which can lead to portal vein thrombosis were excluded before making the diagnosis. Taking into account that venous thromboembolism is the most thrombotic event reported in patients with COVID-19 disease, venous thrombosis at unusual sites like portal vein should be considered.
The exact pathophysiology of the increased prevalence of thromboembolic events in COVID-19 disease still remains unclear. COVID-19 disease shares multiple similarities with other well-defined inflammatory states such as sepsis wherein simultaneous rise in pro and anti-inflammatory cytokines are seen [14]. There is evidence of complement activation in COVID-19 disease by direct endothelial infection. The

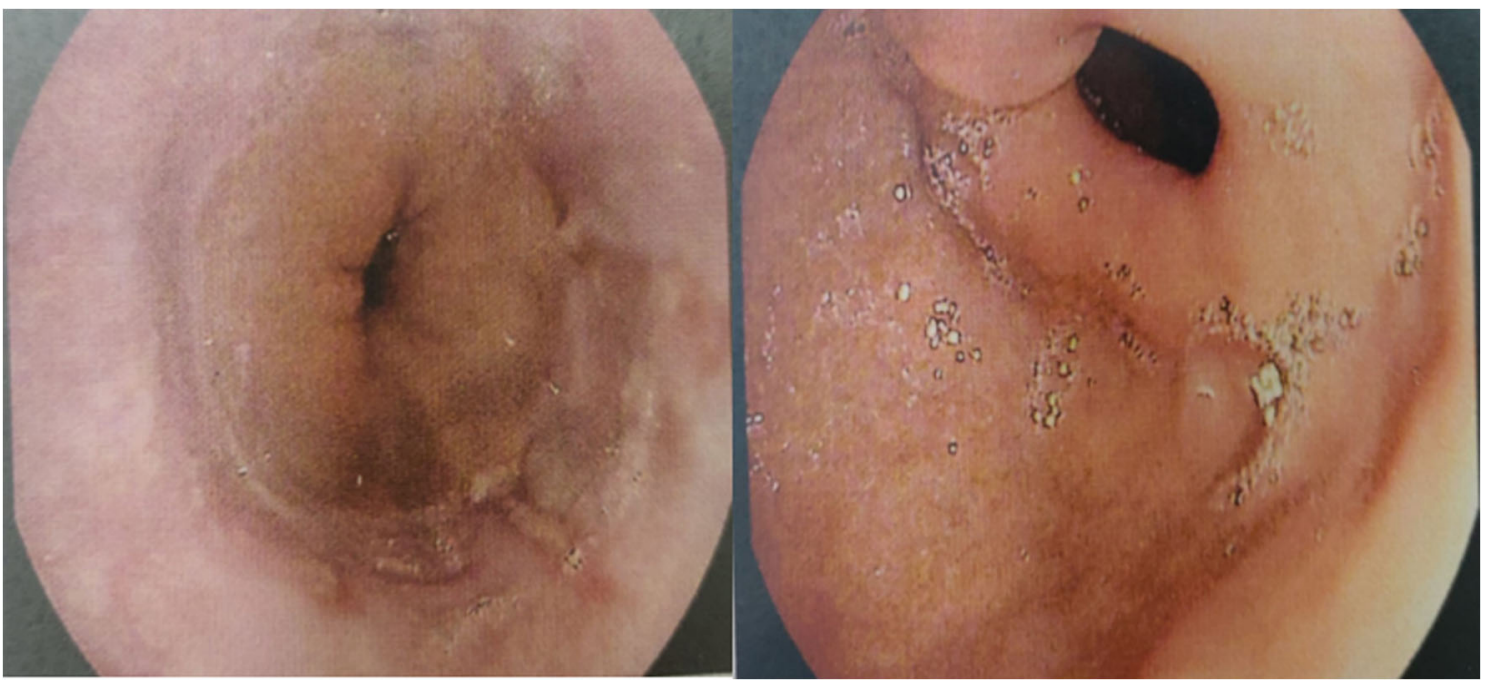

Fig. 2 Upper gastrointestinal endoscopy 
attachment of the viruses to the endothelial surfaces via angiotensin-converting enzyme receptor leads to the lymphocytic endotheliitis, which induces the release of anaphylatoxin C5a [15]. This leads to complement activation which further activates the coagulation system and thereby propagating a prothrombotic state. It also causes interferon-1 and prothrombotic genes overexpression [16]. Therefore, it seems that in keeping with Virchow's triad, thrombosis in COVID19 infection is driven both by the activation of coagulation factors and endothelium involvement. In addition to the factors mentioned above, this thrombotic risk is compounded by hypoxia that typically manifests in severe respiratory COVID19 cases [17].

The management of these patients involves optimal anticoagulation therapy. Both low molecular weight heparin (LMWH) and unfractionated heparin have been successfully used in these patients both therapeutically and prophylactically [18]. Current strategies of anticoagulation in these patients are heavily influenced by empirical institutional protocols, observational reports, and case series as there is absence of well-conducted clinical trials.

\section{Conclusion}

COVID-19 disease is a markedly prothrombotic state. When reviewing patients with COVID-19 infection presenting with abdominal pain, clinicians should consider portal vein thrombosis in their differential diagnosis. While this is a rare complication of COVID-19 infection, clinical threshold for performing liver imaging must be lower in a proper clinical scenario to avoid missing this reversible and rare complication of COVID-19 infection. Also, one should not expect to see grossly deranged liver function tests with acute portal vein thrombosis.

Code Availability Not applicable.

Author Contribution All the authors have been involved in the review of the case report.

Data Availability Not applicable.

\section{Declarations}

Ethics Approval Not applicable.

Consent to Participate Not applicable.

Consent for Publication Taken from the patient.

Conflict of Interest The authors declare no competing interests.

\section{References}

1. Rotzinger DC, Beigelman-Aubry C, von Garnier C, Qanadli SD. Pulmonary embolism in patients with COVID-19: time to change the paradigm of computed tomography. Thromb Res. 2020;190:58-9.

2. Casey K, Iteen A, Nicolini R, et al. COVID-19 pneumonia with hemoptysis: acute segmental pulmonary emboli associated with novel coronavirus infection. Am J Emerg Med. 2020;38:1544.e1-3.

3. Huang C, Wang Y, Li X, Ren L, Zhao J, Hu Y, et al. Clinical features of patients infected with 2019 novel coronavirus in Wuhan, China. Lancet. 2020;395:497-506.

4. Cheung KS, Hung IFN, Chan PPY, Lung KC, Tso E, Liu R, et al. Gastrointestinal manifestations of SARS-CoV-2 infection and virus load in fecal samples from a Hong Kong cohort: systematic review and meta-analysis. Gastroenterology. 2020;159:81-95.

5. Valla D, Casadevall N, Huisse MG, et al. Etiology of portal vein thrombosis in adults. A prospective evaluation of primary myeloproliferative disorders. Gastroenterology. 1988;94:1063-g.

6. Helms J, Tacquard C, Severac F, et al. High risk of thrombosis in patients with severe SARS-CoV-2 infection: a multicenter prospective cohort study. Intensive Care Med. 2020;46:1089-98.

7. Klok FA, Kruip M, van der Meer NJM, et al. Incidence of thrombotic complications in critically ill ICU patients with COVID-19. Thromb Res. 2020;191:145-7.

8. Klok FA, Kruip M, van der Meer NJM, et al. Confirmation of the high cumulative incidence of thrombotic complications in critically ill ICU patients with COVID-19: an updated analysis. Thromb Res. 2020;191:148-50.

9. La Mura V, Artoni A, Martinelli I, Rossio R, Gualtierotti R, Ghigliazza G, et al. Acute portal vein thrombosis in SARS-CoV-2 infection: a case report. Am J Gastroenterol. 2020 Jul 1;115(7): 1140-2. https://doi.org/10.14309/ajg.0000000000000711.PMID: 32618673;PMCID:PMC7273956.

10. Ofosu A, Ramai D, Novikov A, Sushma V. Portal vein thrombosis in a patient with COVID-19. Am J Gastroenterol. 2020 Sep;115(9): 1545-6. https://doi.org/10.14309/ajg.0000000000000781.PMID: 32694290;PMCID:PMC7396211.

11. Franco-Moreno A, Piniella-Ruiz E, Montoya-Adarraga J, BallanoFranco C, Alvarez-Miguel F, Peinado-Martinez C, et al. Portal vein thrombosis in a patient with COVID-19. Thromb Res. 2020 Oct;194:150-2. https://doi.org/10.1016/j.thromres.2020.06.019.

12. Jafari SH, Naseri R, Khalili N, Haseli S, Bahmani M. Portal vein thrombosis associated with COVID-19: points to consider. BJR Case Rep. 2020 Jul 24;6(3):20200089. https://doi.org/10.1259/ bjrcr.20200089.PMID:32922855;PMCID:PMC7465749.

13. Borazjani R, Seraj SR, Fallahi MJ, Rahmanian Z. Acute portal vein thrombosis secondary to COVID-19: a case report. BMC Gastroenterol. 2020 Nov 19;20(1):386. https://doi.org/10.1186/ s12876-020-01518-2.PMID:33213374;PMCID:PMC7675382.

14. Yang Y SC, Li J, Yuan J, Yang M, et al. Exuberant elevation of IP10, MCP-3 and IL-1 ra during SARS-CoV-2 infection is associated with disease severity and fatal outcome. medRxiv 2020.

15. Gao T HM, Zhang X, Li H, Zhu L, et al. Highly pathogenic coronavirus $\mathrm{N}$ protein aggravates lung injury by MASP-2-mediated complement overactivation. medRxiv 2020.

16. Sharma A, Garcia G, Arumugaswami V, Svendsen CN. Human iPSC-derived cardiomyocytes are susceptible to SARS-CoV-2 infection. Cell Rep Med. 2020;1(4):100052.

17. Gupta N, Zhao YY, Evans CE. The stimulation of thrombosis by hypoxia. Thromb Res. 2019;181:77-83.

18. Thachil J, Tang N, Gando S, et al. Type and dose of heparin in COVID-19. J Thromb Haemost. 2020.

Publisher's Note Springer Nature remains neutral with regard to jurisdictional claims in published maps and institutional affiliations. 